Original Article

\title{
Effect of sensorimotor stimulation on chronic stroke patients' upper extremity function: a preliminary study
}

\author{
Eun-Ji Go, OT, BSc ${ }^{1)}$, SANG-HeOn LeE, OT, $\mathrm{PhD}^{2)^{*}}$ \\ 1) Department of Occupational Therapy, Soonchunhyang University Graduate School, \\ Republic of Korea \\ 2) Department of Occupational Therapy, College of Medical Sciences, Soonchunhyang University: \\ Soonchunhyang ro 22, Shinchang-myeon, Asan-si, Chungcheongnam-do 336-745, Republic of Korea
}

\begin{abstract}
Purpose] The aim of this study was to investigate the effect of an intensive sensorimotor stimulation program on the motor function of chronic hemiparetic patients. [Subjects and Methods] The subjects were three chronic stroke patients whose sensory function was intact, who had Mini-Mental State Examination - Korean version scores of more than 26, and manual muscle test scores of more than fair for affected shoulder and elbow. The research design was an A-B single subject experimental design. The intervention consisted of 4 baselines phase sessions, and 12 sensorimotor stimulation phase sessions. The sensory and motor stimulation was performed for 30 minutes per session. The efficacy of the program was evaluated by the Box and Block test, and the 10-second test. [Results] Box and Block test and 10-second test scores of each subject improved after the 8 weeks intervention. [Conclusion] The intensive sensorimotor stimulation program for the upper extremity may be an efficacious method for improving the function of the affected limb of chronic stroke patients.

Key words: Chronic stroke, Sensorimotor stimulation, Upper extremities function
\end{abstract}

(This article was submitted Jun. 20, 2016, and was accepted Aug. 9, 2016)

\section{INTRODUCTION}

Stroke is a transient or permanent alteration in the function of one or several areas of the brain, and is a consequence of a circulatory disorder. Patients who have suffered a stroke usually present motor and sensory impairments, cognitive and perceptive deficits, and emotional disorders ${ }^{1}$. About $55-75 \%$ of survivors 6 months after stroke cannot use the affected hand for their activities of daily living. Thus, Stroke has an important impact on their quality of life $\mathrm{e}^{2,3)}$.

Neuroscientific evidence supports the premise that the sensory receptors in the fingers generate action potentials that communicate with multiple neural networks and specific neurons in the cerebral cortex that correspond with the hand and face $^{4-9)}$. There is also strong evidence that the development of sensorimotor skills in the hands results in substantial changes (expansion) in the corresponding regions of the cortex ${ }^{10,11)}$.

Recent studies have indicated there is a relation between sensory and motor functions which influences the recovery of motion after a stroke ${ }^{3)}$. Sensorimotor training is popularly applied as a preventive or rehabilitative exercise method in various rehabilitation settings ${ }^{12}$. Sensorimotor training uses some combination of sensory input and motor activities to facilitate the expected normal motor response and promote motor skill development ${ }^{13)}$. Sensorimotor stimulation is designed to produce an adaptive response ${ }^{14)}$, which is defined as behavior of a more advanced, organized, flexible or productive nature than that occurring before the stimulation ${ }^{15}$. The goals of sensorimotor stimulation are to initiate desired movement, facilitate weak movement and inhibit undesired movement for purposeful and coordinated motor behavior ${ }^{16)}$. In sensorimotor stimulation

\footnotetext{
*Corresponding author. Sang-Heon Lee (E-mail: sangheon@sch.ac.kr)

(C)2016 The Society of Physical Therapy Science. Published by IPEC Inc.

This is an open-access article distributed under the terms of the Creative Commons Attribution Non-Commercial No Derivatives (by-nc-nd) License $<$ http://creativecommons.org/licenses/by-nc-nd/4.0/>.
} 
therapy, interventions rely on the use of exterocepters and proprioceptors, but not interoceptors, since it is thought that behaviors are learned through exteroceptive and proprioceptive stimulation. There are various modalities for stimulating exteroceptors to enhance sensorimotor function ${ }^{15,17)}$. Nevertheless, actual interventions in clinical settings that demonstrate restoration of motor function are those that focus on repetitive task-specific practice with feedback on performance, so little attention has been given to interventions involving sensorimotor stimulation, particularly in chronic hemiparesis ${ }^{18)}$. Therefore, it may be of interest to develop new protocols for sensorimotor stimulation ${ }^{1)}$.

The aim of this study was to investigate the effectiveness of an intensive sensorimotor stimulation program for the upper limb of patients with chronic hemiparesis due to stroke.

\section{SUBJECTS AND METHODS}

This study was a preliminary study which was performed to direct future research, and to investigate the effectiveness of a sensorimotor stimulation program. Ethical approval was granted from the Institutional Review Board on Human Subjects Research and Ethics Committees, Soonchunhyang University, Cheonan, Korea. All participants provided their informed consent before participating in this study. The participants were chronic stroke patients whose sensory functions were intact, who had Mini-Mental State Examination - Korean version (MMSE-K) scores of more than 26, and manual muscle test (MMT) scores of more than fair for the affected shoulder and elbow.

Two females and one male participated in this study (Table 1). The participants were recruited from a regional rehabilitation hospital and their mean age was 58.3 years. The affected side of two the subjects was the right side. Informed consent was obtained from the subjects before starting the evaluations and the interventions. The onset time of stoke was more than 6 months earlier and the participants were receiving conventional rehabilitation therapy for one hour at a rehabilitation center.

The research design was an A-B single subject experiment design. The intervention was performed twice a week and consisted of 5 baseline sessions of 5 minutes per session, followed by 12 treatment sessions lasting 30 minutes with evaluation sessions after sensorimotor stimulation. Each rehabilitation session was performed with the assistance of one therapist and was divided in two phases, a passive hand mobilization phase and a sensory stimulation phase. During the first 10 minutes, the patients' hand were prepared with specific movements to reduce muscle tone, followed by passive mobilization of the metacarpophalangeal joints and lengthening of thenar and hypothenar muscle groups and interossei muscles. Then, passive sensory training involving vibration and proprioception was conducted for 20 minutes. Vibration was provided by vibrating toothbrushes to stimulate the intrinsic and extrinsic muscles of the hand at a frequency of $127 \mathrm{~Hz}$. Then, proprioceptive training was performed. For the proprioceptive training of the wrist joint, a therapist positioned the subject's affected wrists at specific angles of motion ( 0,30 , and 60 degrees flexion and 30, and 60 degrees of extension), and the subjects were asked to report the wrist angle. Next, the finger joint of affected hand were moved to specific positions, and the subjects imitated the position using their non-affected hand.

The Box and Block test (BBT) and 10-second test were used to evaluate hand function in this study. The BBT measures unilateral gross manual dexterity and is frequently used in research and rehabilitation involving both children and adults. This test consists of moving, one by one, the greatest number of blocks as possible from one compartment of a box to another of equal size, within 60 seconds. A 6-month test-retest reliability study was done ( $\mathrm{p}=0.98$ for the right hand and 0.92 for the left) and concomitant validity was measured using the Minnesota Rate Manipulation Test which gave a result of $r=0.91$. 10 -second test consists of three types of tests. The examinee performs three types of hand-finger movements (the finger individual movement test: FIMT; the hand pronation and supination test: HPST; and the finger tapping test: FTT) as quickly as possible for 10 seconds, and the number of movements is counted. The intraclass correlation coefficients of the 10 second test ranged from 0.74 to 0.84 and the concomitant validity was measured using the Fugl-Meyer Motor Function Assessment which gave a result of $\mathrm{r}=0.88$.

In this study, the descriptive data analysis was used to analyze the results.

\section{RESULTS}

In the BBT (Table 2), participant A's mean score increased from 62.0 to 67.7 on the non-affected side, and from 39.0 to 47.3 on the affected side; participant B's mean score increased from 55.8 to 64.4 on the non-affected side, and from 26.3 to 34.1 on the affected side; participant C's mean score increased from 33.3 to 40.0 on the non-affected side, and from 18.3 to 28.6 on the affected side.

In the 10-second test (Table 3), participant A's mean score increased from 3.0 to 4.2 in FIMT, from 11.3 to 14.3 in HPST, and from 25.0 to 32.2 in FTT; participant B's score mean increased from 2.0 to 3.4 in FIMT, form 7.0 to 10.6 in HPST, and from 26.3 to 34.1 in FTT; and participant C's score mean increased from 2.0 to 2.2 in FIMT, from 9.3 to 10.4 in HPST, and from 20.3 to 27.5 in FTT. 
Table 1. General characteristics of the subjects

\begin{tabular}{lcllccc}
\hline & Gender & Diagnosis & Affected side & Onset time & Age (yrs) K-MMSE \\
\hline Participant A & F & Lt. MCA infarction & Rt. hemiplegia & 24 months & 43 & 29 \\
Participant B & M & Lt. MCA infarction & Rt. hemiplegia & 18 months & 52 & 28 \\
Participant C & F & Rt. BG intracerebral hemorrage & Lt. hemiplegia & 10 months & 80 & 26 \\
\hline
\end{tabular}

*Korean Mini-Mental State Examination

Table 2. Box and Block test scores (value $=$ number)

\begin{tabular}{|c|c|c|c|c|c|c|c|c|c|c|c|c|c|c|c|c|c|}
\hline & & \multicolumn{4}{|c|}{ Baseline } & \multicolumn{12}{|c|}{ Intervention } \\
\hline & & 1 & 2 & 3 & 4 & 1 & 2 & 3 & 4 & 5 & 6 & 7 & 8 & 9 & 10 & 11 & 12 \\
\hline \multirow{2}{*}{ Participant A } & $\mathrm{AS}^{*}$ & 40 & 41 & 38 & 37 & 44 & 44 & 44 & 45 & 45 & 50 & 44 & 47 & 51 & 48 & 53 & 53 \\
\hline & NAS** & 61 & 67 & 62 & 58 & 63 & 65 & 64 & 68 & 68 & 71 & 67 & 68 & 68 & 68 & 71 & 71 \\
\hline \multirow{2}{*}{ Participant B } & AS & 27 & 21 & 24 & 33 & 31 & 28 & 29 & 32 & 33 & 33 & 36 & 33 & 36 & 36 & 40 & 42 \\
\hline & NAS & 60 & 59 & 50 & 54 & 58 & 59 & 55 & 63 & 62 & 61 & 63 & 63 & 65 & 68 & 76 & 80 \\
\hline \multirow{2}{*}{ Participant $\mathrm{C}$} & AS & 12 & 20 & 19 & 22 & 19 & 25 & 26 & 25 & 31 & 27 & 30 & 31 & 31 & 32 & 33 & 33 \\
\hline & NAS & 23 & 33 & 33 & 44 & 30 & 28 & 43 & 40 & 42 & 41 & 42 & 41 & 42 & 43 & 43 & 45 \\
\hline
\end{tabular}

*Affected side, **Non-affected side

Table 3. 10-second test scores of affected side (value $=$ number)

\begin{tabular}{|c|c|c|c|c|c|c|c|c|c|c|c|c|c|c|c|c|c|}
\hline & & \multicolumn{4}{|c|}{ Baseline } & \multicolumn{12}{|c|}{ Intervention } \\
\hline & & 1 & 2 & 3 & 4 & 1 & 2 & 3 & 4 & 5 & 6 & 7 & 8 & 9 & 10 & 11 & 12 \\
\hline \multirow{3}{*}{ Participant A } & FIMT* & 3 & 3 & 3 & 3 & 3 & 3 & 3 & 4 & 4 & 4 & 4 & 4 & 5 & 5 & 5 & 6 \\
\hline & HPST** & 12 & 12 & 11 & 10 & 11 & 12 & 13 & 13 & 14 & 13 & 15 & 15 & 15 & 16 & 17 & 17 \\
\hline & $\mathrm{FTT}^{* * *}$ & 27 & 25 & 23 & 25 & 27 & 26 & 28 & 29 & 32 & 31 & 33 & 35 & 36 & 36 & 37 & 36 \\
\hline \multirow{3}{*}{ Participant B } & FIMT & 2 & 2 & 2 & 2 & 2 & 3 & 3 & 3 & 3 & 3 & 4 & 4 & 4 & 4 & 4 & 4 \\
\hline & HPST & 7 & 7 & 7 & 7 & 8 & 8 & 9 & 10 & 9 & 11 & 11 & 11 & 11 & 12 & 13 & 14 \\
\hline & FTT & 19 & 23 & 18 & 22 & 24 & 25 & 29 & 28 & 29 & 29 & 29 & 29 & 30 & 31 & 33 & 34 \\
\hline \multirow{3}{*}{ Participant C } & FIMT & 2 & 2 & 2 & 2 & 2 & 2 & 2 & 2 & 2 & 2 & 2 & 2 & 2 & 2 & 3 & 3 \\
\hline & HPST & 8 & 10 & 10 & 9 & 9 & 10 & 9 & 11 & 10 & 10 & 11 & 10 & 11 & 11 & 11 & 12 \\
\hline & FTT & 16 & 21 & 18 & 26 & 20 & 25 & 28 & 25 & 23 & 25 & 29 & 30 & 31 & 31 & 30 & 33 \\
\hline
\end{tabular}

*FIMT: Finger Individual Movement Test, **HPST: Hand Pronation and Supination Test, ***FTT: Finger Tapping Test

\section{DISCUSSION}

Sensory stimulation is required for accurate motor performance and more effective motor learning. Caliandro et al. showed the effectiveness of repetitive focal muscle vibration in the treatment of upper limb spasticity ${ }^{18)}$, and the Functional Ability Scale of the Wolf Motor Function test score also showed there was significant improvement. Recently, Sim et al. showed that sensory stimulation enhanced performance of hand tasks in chronic stroke patients ${ }^{19}$.

Passive range of motion therapy helps maintain range and flexibility and temporarily reduces hypertonia or resistance to passive movement. Hesse et al. examined with 8 stroke patients and found passive movement was as assessed by the Fugl-Meyer test ${ }^{20)}$. In Volpe's study, repetitive passive ROM exercise influenced the motor function $(\mathrm{p}=0.01)$ and power in the trained shoulder and elbow $(\mathrm{p}=0.0001)^{21)}$.

The main finding of the present study was that somatosensory stimulation of the paretic hand immediately enhanced the hand function. Despite substantial advances in the development of more effective training protocols, the functional recovery process is accompanied by long-term motor disability ${ }^{22}$. Somatosensory input is required for learning and performance of skillful motor tasks ${ }^{23)}$. When this input is reduced or absent, poor motor behavior ensures ${ }^{24)}$. It is not surprising that after stroke, patients with somatosensory deficits suffer more persistent motor impairment than those without such deficits ${ }^{25}$.

In this study, we found that the scores of the BBT and 10-second test was increased after training with sensorimotor stimulation. These findings suggest that sensoimotor training is an effective therapeutic approaches which enhances the upper extremity function of chronic stroke patients. In most previous investigations, sensorimotor training included active movement training with sensory stimulation, but in this study only passive movement with sensory stimulation was conducted. 
Therapist need to consider passive movement with sensory stimulation as an options in stroke rehabilitation.

Some limitations of this study have to be considered, including the small sample size and the absence of and evaluating of long-term effects. The same study protocol should be performed using a larger population and a long observation time.

\section{ACKNOWLEDGEMENT}

This work was supported by the Soonchunhyang University Research Fund.

\section{REFERENCES}

1) de Diego C, Puig S, Navarro X: A sensorimotor stimulation program for rehabilitation of chronic stroke patients. Restor Neurol Neurosci, 2013 , 31: 361-371. [Medline]

2) Wolf SL, Winstein CJ, Miller JP, et al. EXCITE Investigators: Effect of constraint-induced movement therapy on upper extremity function 3 to 9 months after stroke: the EXCITE randomized clinical trial. JAMA, 2006, 296: 2095-2104. [Medline] [CrossRef]

3) Doyle S, Bennett S, Fasoli SE, et al.: Interventions for sensory impairment in the upper limb after stroke. Cochrane Database Syst Rev, 2010, 6: CD006331. [Medline]

4) Bangert M, Schlaug G: Specialization of the specialized in features of external human brain morphology. Eur J Neurosci, 2006, 24: 1832-1834. [Medline] [CrossRef]

5) Kaas JH: Plasticity of sensory and motor maps in adult mammals. Annu Rev Neurosci, 1991, 14: 137-167. [Medline] [CrossRef]

6) Kaas JH: Topographic maps are fundamental to sensory processing. Brain Res Bull, 1997, 44: 107-112. [Medline] [CrossRef]

7) Kaas JH: The future of mapping sensory cortex in primates: three of many remaining issues. Philos Trans R Soc Lond B Biol Sci, 2005, 360: 653-664. [Medline] [CrossRef]

8) Merzenich MM, Jenkins WM: Reorganization of cortical representations of the hand following alterations of skin inputs induced by nerve injury, skin island transfers, and experience. J Hand Ther, 1993, 6: 89-104. [Medline] [CrossRef]

9) Merzenich M, Wright B, Jenkins W, et al.: Cortical plasticity underlying perceptual, motor, and cognitive skill development: implications for neurorehabilitation. Cold Spring Harb Symp Quant Biol, 1996, 61: 1-8. [Medline] [CrossRef]

10) Burton H, Sinclair RJ, McLaren DG: Cortical activity to vibrotactile stimulation: an fMRI study in blind and sighted individuals. Hum Brain Mapp, 2004, 23: 210-228. [Medline] [CrossRef]

11) Recanzone GH, Merzenich MM, Dinse HR: Expansion of the cortical representation of a specific skin field in primary somatosensory cortex by intracortical microstimulation. Cereb Cortex, 1992, 2: 181-196. [Medline] [CrossRef]

12) McCaskey MA, Schuster-Amft C, Wirth B, et al.: Effects of postural specific sensorimotor training in patients with chronic low back pain: study protocol for randomised controlled trial. Trials, 2015, 16: 571. [Medline] [CrossRef]

13) Caiten N, Polatajko H: Evidence brief for occupational therapy intervention for sensory-motor problems. AOTA Evidenced-Based Practice Review, 2008.

14) Ayres AJ: Sensory integration and learning disorders, LA. Western Psychological, 1980.

15) Farber SD: Neurorehabilitation: a multisensory approach. Philadelphia: WB Saunders, 1982.

16) Regina E: Sensory stimulation techniques. Am J Nurs, 1966, 66: 281-286. [Medline]

17) Heiniger MC, Randolph SL: Neurophysiological concepts in human behavior. St. Louis: The C. V. Mosby Co., 1981.

18) Caliandro P, Celletti C, Padua L, et al.: Focal muscle vibration in the treatment of upper limb spasticity: a pilot randomized controlled trial in patients with chronic stroke. Arch Phys Med Rehabil, 2012, 93: 1656-1661. [Medline] [CrossRef]

19) Sim SM: Effects of sensory stimulation theapy on upper limb's kinematic data in patients with post-stroke hemiparesis: a randomized cross-over pilot trial. Kor J Neural Rehabil. 2014, 4: 14-20.

20) Hesse S, Kuhlmann H, Wilk J, et al.: A new electromechanical trainer for sensorimotor rehabilitation of paralysed fingers: a case series in chronic and acute stroke patients. J Neuroeng Rehabil, 2008, 5: 21. [Medline] [CrossRef]

21) Volpe BT, Ferraro M, Lynch D, et al.: Robotics and other devices in the treatment of patients recovering from stroke. Curr Atheroscler Rep, 2004, 6: 314-319. [Medline] [CrossRef]

22) Gresham GE, Fitzpatrick TE, Wolf PA, et al.: Residual disability in survivors of stroke-the Framingham study. N Engl J Med, 1975, 293: 954-956. [Medline] [CrossRef]

23) Pavlides C, Miyashita E, Asanuma H: Projection from the sensory to the motor cortex is important in learning motor skills in the monkey. J Neurophysiol, 1993, 70: 733-741. [Medline]

24) Rothwell JC, Traub MM, Day BL, et al.: Manual motor performance in a deafferented man. Brain, 1982, 105: 515-542. [Medline] [CrossRef]

25) Smania N, Montagnana B, Faccioli S, et al.: Rehabilitation of somatic sensation and related deficit of motor control in patients with pure sensory stroke. Arch Phys Med Rehabil, 2003, 84: 1692-1702. [Medline] [CrossRef] 